\section{P281 DOCUMENTATION OF TEMPERATURE, BLOOD SUGAR AND WEIGHT ON INFANTS PRESENTING TO THE EMERGENCY DEPARTMENT AND THEIR IMPACT}

Khalid Abdalla*, Cormac Duff, Michael Sweeney. Sligo University Hospital, Sligo, Ireland

10.1136/archdischild-2019-epa.631

Introduction The Irish Children's Triage System (ICTS) is a child-specific triage tool that should be followed for the prioritization and assessment of pediatric patients presenting to an Emergency Department in Ireland. It categorizes patient into 5 different groups depending on Presenting problem, General appearance, Physiological findings, Age and Significant past medical history that may impact on the current attendance, Red being the most urgent one and patients need to be seen immediately, orange is category 2 and should be seen in less than 10 minutes. Vital signs play a major role on deciding how the patient will be categorized. Sligo University Hospital guidelines advise to check temperature, $\mathrm{BM}$ and weight on all infants presenting to ED. Temperature and BM can change the Triage category if one or both were abnormal while weight should be taken for doses to be given and developmental assessment.

Aims The aim of this study is to review current practice in SUH and assess if this follows current guidelines, also check what improvement can be made if these guidelines are not being met.

Methods Over a period of 2 weeks, 58 Infants presented to the Emergency Department of Sligo University Hospital, Data was collected from triage and ED notes. All infants were included in the study regardless of the presentation. The study checked whether temperature, BM and weight were checked and recorded or not by both Doctors and Nurses.

Results 58 patients were identified, regarding nursing triage notes, temperature was documented in $86.2 \%$, BM was documented in $82.7 \%$, and weight was the least to be documented with $60 \%$.

Doctor's Documentation had lower percentages than triage notes with temperature and BM being documented in 51\% and $43.1 \%$ respectively, weight documentation was very low by doctors with only $22.4 \%$. Reasons for not documenting any of the parameters were not clearly identified on the notes.

conclusion From the results above it's evident that documentation of these specific parameters has been significantly higher when done by triage nurses.

Documentations should be improved by responsible physicians.

It's important to always document temperature and BM as patient triage category can be changed depending on them, weight is important when infant development and follow up is being considered.

Presentation and teaching should be done regarding the importance of documentation of these parameters.

A repeat audit should be carried out in order to see if changes are being implemented.

\section{P282 \\ THE CURIOUS CASE OF THE BOY WHO COULDN'T WALK}

Tadgh Moriarty*, Brendan McCann. University Hospital Waterford, Waterford, Ireland

10.1136/archdischild-2019-epa.632
Benign Acute Childhood Myositis (BACM) is a rare but selflimiting illness of mid childhood. It tends to present following a viral infection (most often influenza). Predominant clinical features include difficulty walking, and muscle pain. Unfamiliarity with the condition can lead to admission to hospital for costly and invasive investigations.

We present the case of a 5 year old boy who attended a mixed emergency department with apparent inability to walk following a coryzal illness. The initial consultation with his general practitioner led to an urgent transfer to hospital via emergency ambulance due to concern regarding an acute neurological event. However, history and clinical exam coupled with a significantly elevated creatinine kinase serum level allowed an accurate diagnosis of BACM to be made. Appropriate analgesia and advice regarding the natural history of the illness was provided to the parents and he was safely discharged back to the care of hid GP.

This case report aims to highlight the clinical features of BACM, and more importantly those features which are not consistent with the illness, such as absent reflexes and sub acute onset. This case highlights this common presentation of an uncommon illness; the investigations required for diagnosis, and the appropriate disposition of the patient.

Awareness of this diagnosis can prevent unnecessary admission and diagnostics.

\section{P283 PEDIATRICS OF DISASTERS. PREPARATION OF DOCTORS OF THE CITY POLYCLINIC TO WORK IN CONDITIONS OF EMERGENCIES AND TERRORIST ACTS}

${ }^{1,2}$ Konstantin Shapovalov*, 'Larisa Shapovalova, ${ }^{2}$ Sergey Slutsky, ${ }^{2}$ Alexander Chugaev, ${ }^{2}$ Vasily Katorkin. 'State Education Agency of Additional Professional Education of Republic of Komi 'Komi Republican Institute for Development of Education', Syktyvkar, Russian Federation; 'State Budget Agency of Health of the Republic of Komi' Syktyvkar children's clinic ??3', Syktyvkar, Russian Federation

\subsection{6/archdischild-2019-epa.633}

Pediatrics ES is built on the principles of integrity, structure, causality, dynamism and hierarchy.

The subject of the study of pediatrics emergencies are: Factors of catastrophic events on health of children; Mental and psychosomatic disorders; Methods of diagnosis, treatment, prevention and rehabilitation; Forecasting and assessment of consequences of an emergency.

Medical forces of children's clinic may be involved in emergency situations of man-made, natural, economic, criminogenic nature, in case of fire, the threat of an explosion in a medical institution, if it is impossible to provide assistance to the population, during a strike or protest action of medical workers, lack of medicines.

The clinic regularly practiced the actions of doctor in the event of fire or phone call about the laying of explosives and the threat of explosion.

The tasks of a qualified medical nursing team created may vary depending on the conditions of the situation, the number of injured admitted, the time of their delivery, the distance to the nearest medical institutions, and the availability of transportation for evacuation of the affected.

Predictive medical sorting During disasters, children make up about $25 \%$ of the victims and are a priority group in providing assistance. In the conditions of lack of time at the prehospital stage, the method of dynamic observation generally 